\title{
What Is "Chronic" in "Chronic Sleep Reduction" and What Are Its Consequences? A Systematic Scoping Review of the Literature
}

\author{
E.J. De Bruin ${ }^{1,2}$ (D) J. F. Dewald-Kaufmann ${ }^{3,4}$ (D)
}

Accepted: 3 June 2021 / Published online: 4 August 2021

(C) The Author(s) 2021

\begin{abstract}
Purpose of Review In this scoping review, we aimed to (1) provide an overview of chronic sleep reduction by systematically reviewing the existing literature (limited to systematic reviews and meta-analyses), (2) investigate the evidence of the effects of short sleep duration on daytime functioning, and (3) identify research gaps in this field.

Recent Findings The results showed that (1) clear definitions of chronicity of sleep reduction are lacking - none of the included reviews/meta-analyses provided a full definition - and (2) short sleep duration appeared to be related to obesity and diabetes, whereas relations with cardiovascular disease (CVD), cognitive functioning, emotional problems, general health, and mortality, showed either small effects and appeared to be complex (e.g., for CVD and cognitive functioning), or studies were scarce or completely lacking (e.g., mortality in children/adolescents and emotional problems in adults).

Summary Although short or insufficient sleep is highly prevalent and is associated with impaired mental and physical wellbeing, as well as pervasive negative consequences for daytime functioning, the concepts of "chronicity" and "chronic sleep reduction" have not been clearly defined and its effects on health are therefore still largely unknown. Moreover, there are large research gaps concerning studies on the relations between short sleep and health consequences. Further studies are recommended to define and operationalize chronicity of sleep reduction and develop measurements that adequately represent the complexity of the concept.
\end{abstract}

Keywords Sleep reduction $\cdot$ Sleep deprivation $\cdot$ Sleep problems $\cdot$ Daytime consequences

E. J. De Bruin and J. F. Dewald-Kaufmann contributed equally to this work.

This article is part of the Topical Collection on Sleep and Aging

E. J. De Bruin

e.j.debruin@utwente.nl

1 Centre for eHealth and Wellbeing Research, Department of Psychology, Health \& Technology, University of Twente, Enschede, The Netherlands

2 Research Institute of Child Development and Education, Faculty of Social and Behavioral Sciences, University of Amsterdam, Amsterdam, The Netherlands

3 Hochschule Fresenius, University of Applied Sciences, Munich, Germany

4 Department of Psychiatry and Psychotherapy, University Hospital LMU, Munich, Germany

\section{Introduction}

Insufficient and/or inadequate sleep is highly prevalent and is associated with impaired emotional wellbeing [1,2], daytime functioning [3], and physical health [4]. Even the DSM-5 states that (chronic) insomnia does not solely refer to problems with initiating and/or maintaining sleep three times/week for at least 3 months, but also includes impairments in daytime functioning [5]. Sleep deprivation in the laboratory, in which individuals are prohibited to sleep for a defined number of hours and/or nights, is a useful experimental paradigm to study the effects of acute sleep loss under controlled conditions. However, it is less representative of individuals' daily life (i.e., ecological validity), in which reduced sleep is usually experienced over a longer time period (e.g., due to shift work, lifestyle, work load). Furthermore, other influential sleep variables, such as sleep quality and sleep variability, can also best be studied in somebody's natural environment. Partial sleep deprivation can result from fragmented sleep (e.g., in sleep apnea), loss of specific physiological sleep stages, or sleep restriction, which is characterized by the reduced opportunity 
to sleep (e.g., 5, 6, or $7 \mathrm{~h} / 24 \mathrm{~h}$ ) (e.g., [6]). The latter is often referred to as chronic sleep reduction, meaning that sleep is reduced over a longer time, such as several days or even weeks [6] although no clear definition of this concept exists.

In addition to the problem of ecological validity of laboratory research, the comparison of the results from epidemiological studies is often challenged by the use of different definitions for sleep and sleep problems (e.g., sleep reduction, sleep deprivation, sleep problems including sleep disorders, sleep quality, symptoms of sleep disorders, sleep curtailment, short sleep), and by assumptions about the direction of the risk, even though most studies concern cross-sectional data that are not suited for causal inference. Moreover, many of these definitions contain subjective aspects, such as sleep quality and sleepiness. This subjective aspect is also represented in the classification systems of some sleep disorders (e.g., insomnia disorder), but is usually very difficult to quantify or even to define clearly and unidimensional.

In the general population, sleep durations seem to decrease over the years (e.g., [7, 8]) and many studies have addressed the question of the effects of chronically reduced sleep on daytime functioning, both in longitudinal and cross-sectional studies. The "Joint Consensus of the American Academy of Sleep Medicine and Sleep Research Society" concluded that healthy adults should sleep on average 7 or more hours per night, as less sleep is related to negative health outcomes (e.g., weight gain, obesity, diabetes, hypertension, heart disease, stroke, depression, impaired immune function, increased pain, increased risk of accidents, increased risk of death) [9]. However, the concept of chronicity still remains vaguely defined or even undefined, meaning that little is known yet, of how many nights sleep has to be reduced by how many hours, and in which way (e.g., variability, circadian shift) in the context of personal sleep need and satisfaction (e.g., [10]), to negatively affect somebody's daytime functioning. This issue, together with problems of ecological validity and definitions for sleep and sleep problems, leads to the question whether chronic sleep reduction can be defined in such a way that it catches both the quantitative aspect from laboratory studies and the broader phenomenological descriptions and definitions from epidemiological or clinical studies.

In comparison to systematic reviews, scoping reviews aim to map the existing literature, to identify research gaps, to give a broader overview of the literature on a specific topic, and to provide ideas for future research (e.g., [11]). Concerning the topic of this review, two recent studies aimed to summarize the current knowledge concerning the relationship between sleep, developmental aspects, and health in children and adolescents $[12 \bullet, 13]$. Although these studies give a good overview of the research field, both were limited to children and adolescents, and one focused on research of the last 5 years. In order to summarize the existing literature in this field, we, therefore, conducted a scoping review, aiming to provide an overview of chronic sleep reduction by its daytime and health consequences, and by sleep duration and quality, and to identify important research gaps.

\section{Methods}

We conducted a systematic literature search (PsycINFO, Medline, Scopus, Cochrane library) using variations and combinations of the following keywords dependent on the requirements of the databases that were searched: sleep deprivation, insufficient sleep, sleep disruption, systematic review, metaanalysis, suicide, emotions, affect, problem behavior, cognitive functioning, memory, executive functioning (see the addendum for a full search report).

Articles were included in the paper based on the following inclusion criteria: (1) the paper was published in a peerreviewed journal and written in English; (2) the paper was a systematic review or meta-analysis of original studies; (3) the concept of chronic sleep reduction was conceptualized, e.g., a definition of too short or insufficient sleep in quantitative terms was given or the hours of sleep, defined as insufficient sleep in the original studies, were provided; (4) the article addressed a healthy population. Articles were excluded (1) if they were not written in English or not published in a peerreviewed journal; (2) if they were no systematic review/metaanalysis but an original study (e.g., experimental study, crosssectional study); (3) if sleep problems referred to sleep disorders (e.g., insomnia, sleep apnea) or addressed a specific population that is assumed to experience insufficient sleep because of specific circumstances (e.g., pregnant women); (4) if the review focused on participants with a certain disorder, such as psychiatric disorders (e.g., bipolar disorders), dementia, Parkinson's disease, chronic pain, physical illness (e.g., cancer, epilepsy); (5) if the review/meta-analysis included only experimental studies in the laboratory for short-term sleep deprivation or extension. The following characteristics were coded: (1) goals/aims, (2) number and nature of included studies, (3) participants' age, (4) operationalization/definition of chronic sleep reduction (including definition of sleep characteristic, definition of short sleep duration), (5) assessment methods of chronic sleep reduction (e.g., questionnaire, polysomnography), (6) consequences of chronic sleep reduction (e.g., effects on daytime functioning, mental health, physical health), (7) quality assessment of the included studies.

A total of 1726 unique articles were identified with the initial search that was conducted on March 6, 2017. After screening titles and abstracts, 1684 articles were excluded, resulting in 42 articles, which were fully read and coded by two independent researchers (EdB, JDK). From these 42 articles, 27 did not fully meet the in- and exclusion criteria and were excluded, resulting in 15 articles. On June 18, 2018, we updated the search and identified 370 additional unique 
articles. After screening, 19 of these articles were fully read, coded, and discussed, resulting in five additional articles. We repeated this search strategy on May 7, 2019, identified 297 additional unique articles from which 16 were fully read, coded, and discussed, and two additional articles were included. This brought the total number of included articles in the present study to 22 . Figure 1 presents the applied search strategy. Because different reviews/ meta-analyses about similar outcomes often included several of the same original articles, we calculated and reported the unique number of articles and participants (i.e., deleted the overlap). The numbers of articles and participants in Table 1, however, are as the authors originally reported them in the reviews/meta-analyses.
Fig. 1 Database search and article selection
Search with variations of keywords, dependent on the requirements of the databases: sleep deprivation, insufficient sleep, sleep disruption, systematic review, meta-analysis, suicide, emotions, affect, problem behavior, cognitive functioning, memory, executive functioning, health (see the addendum for a full search report).

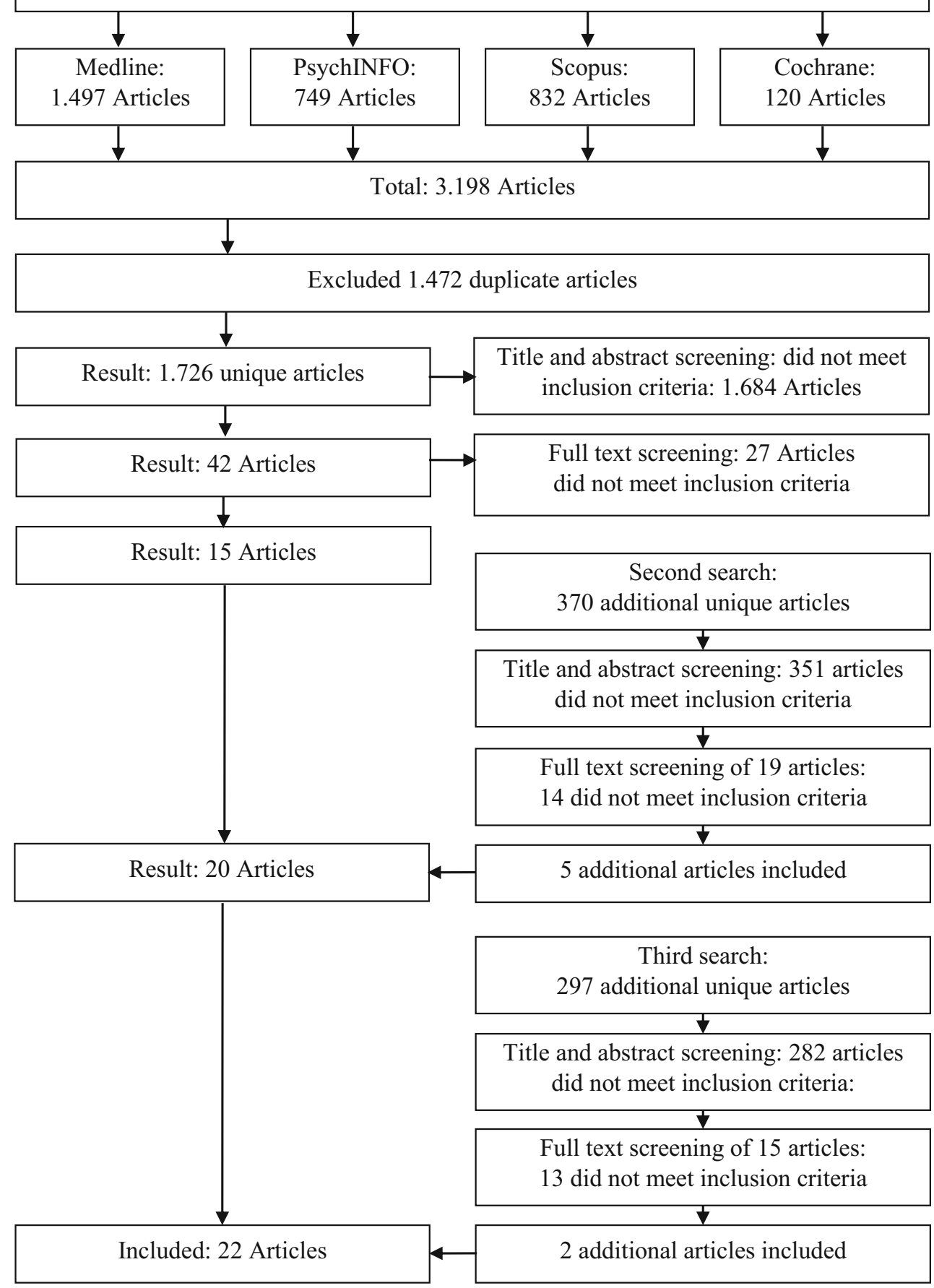




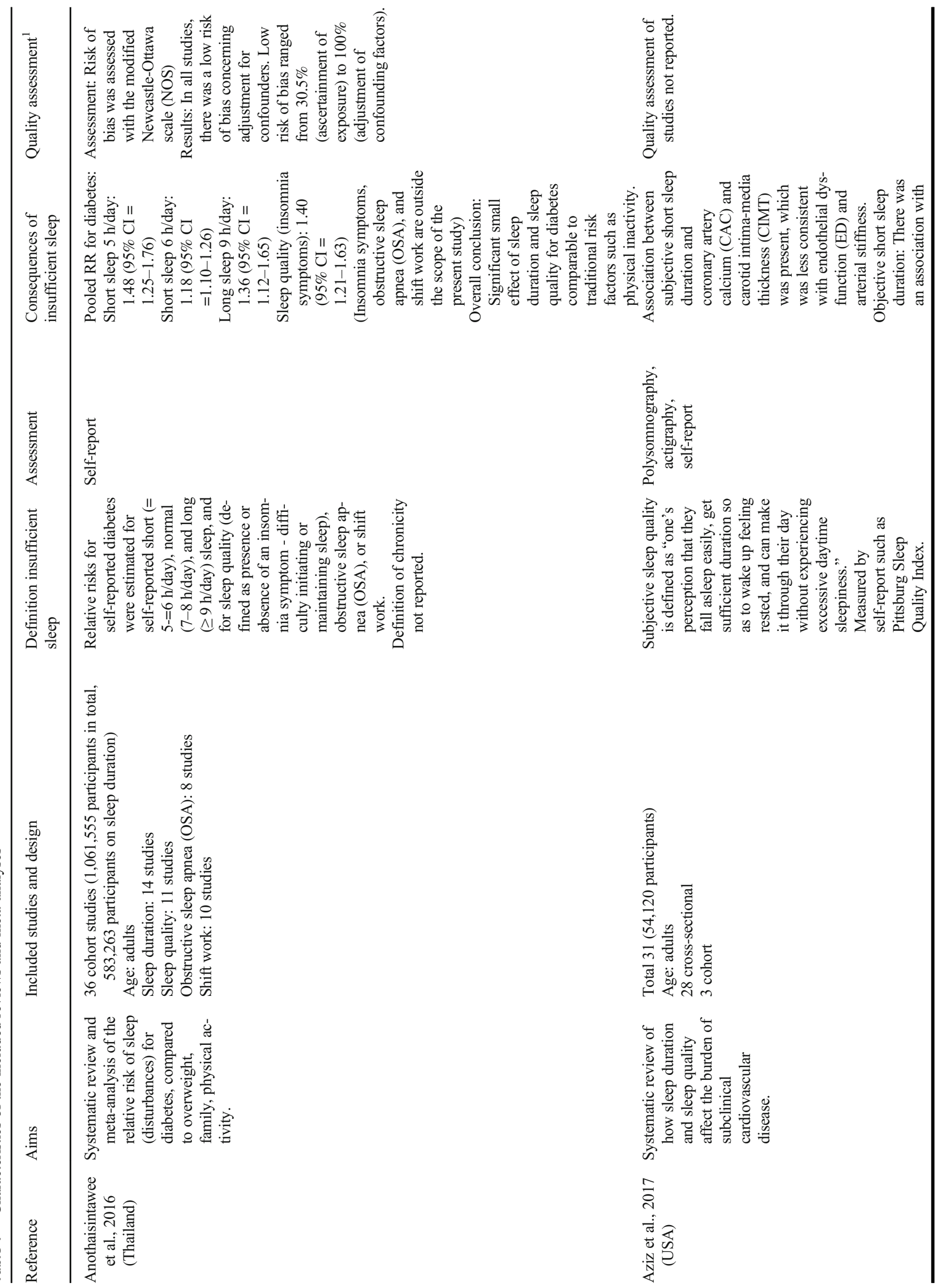




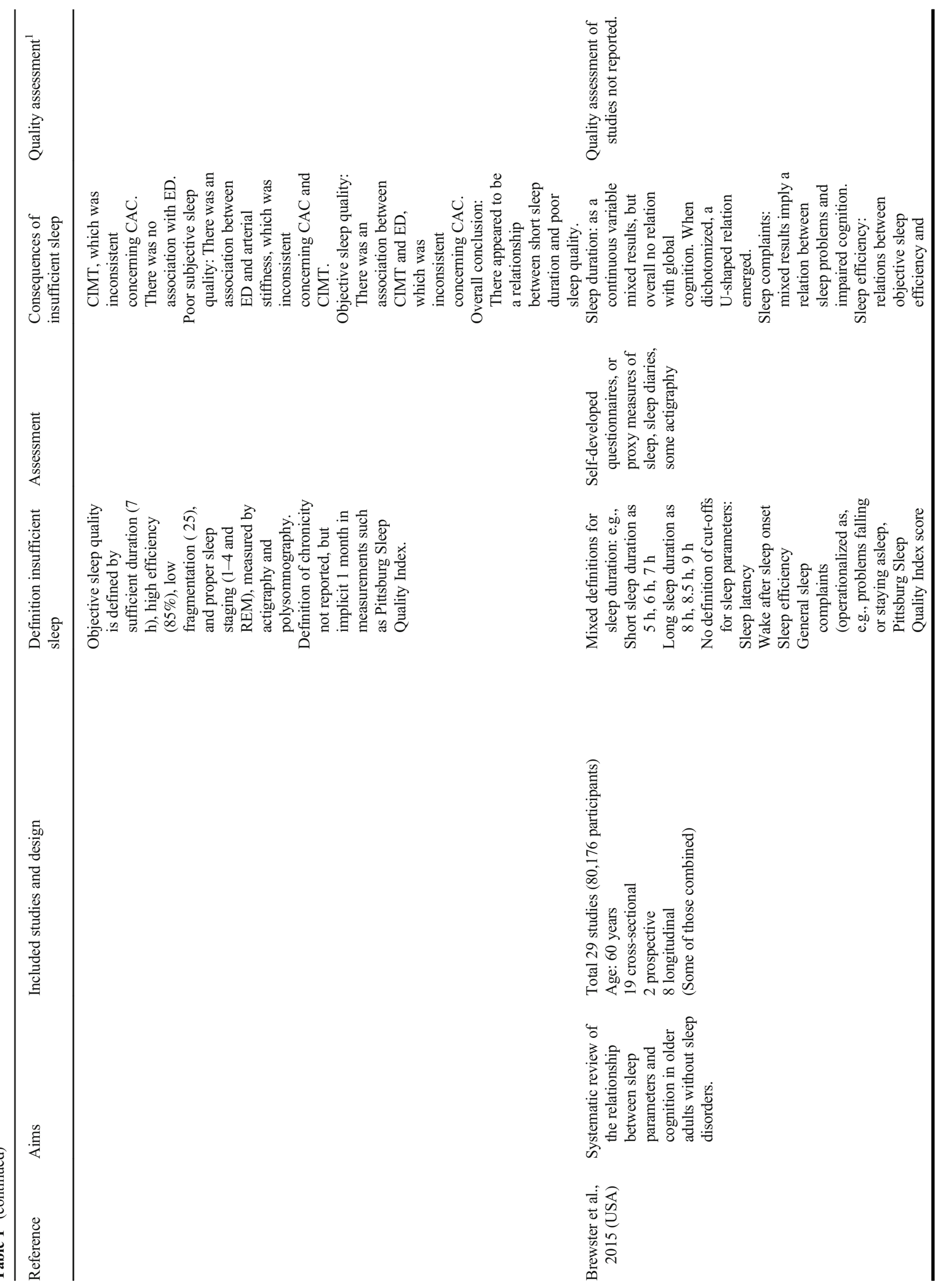




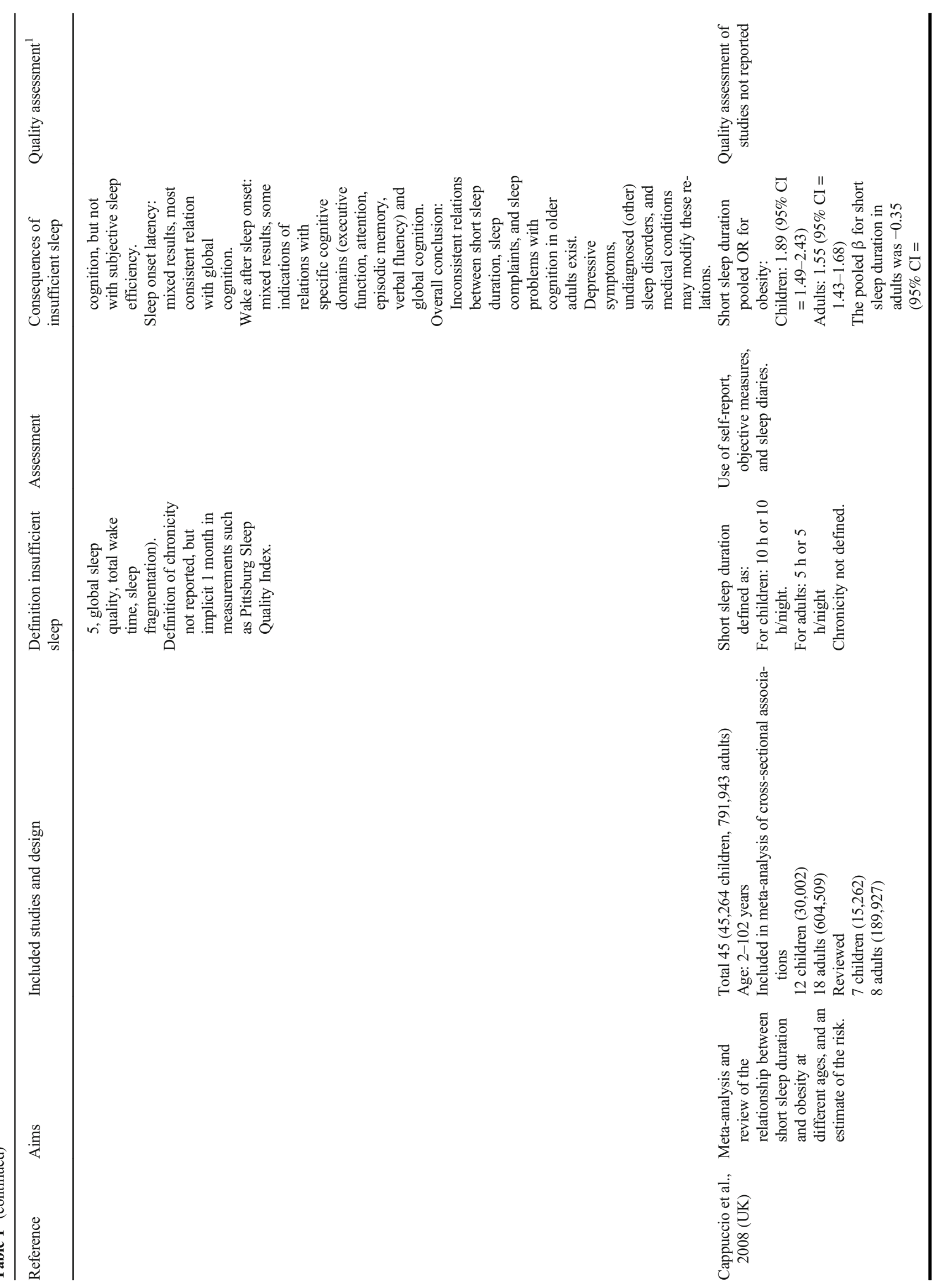




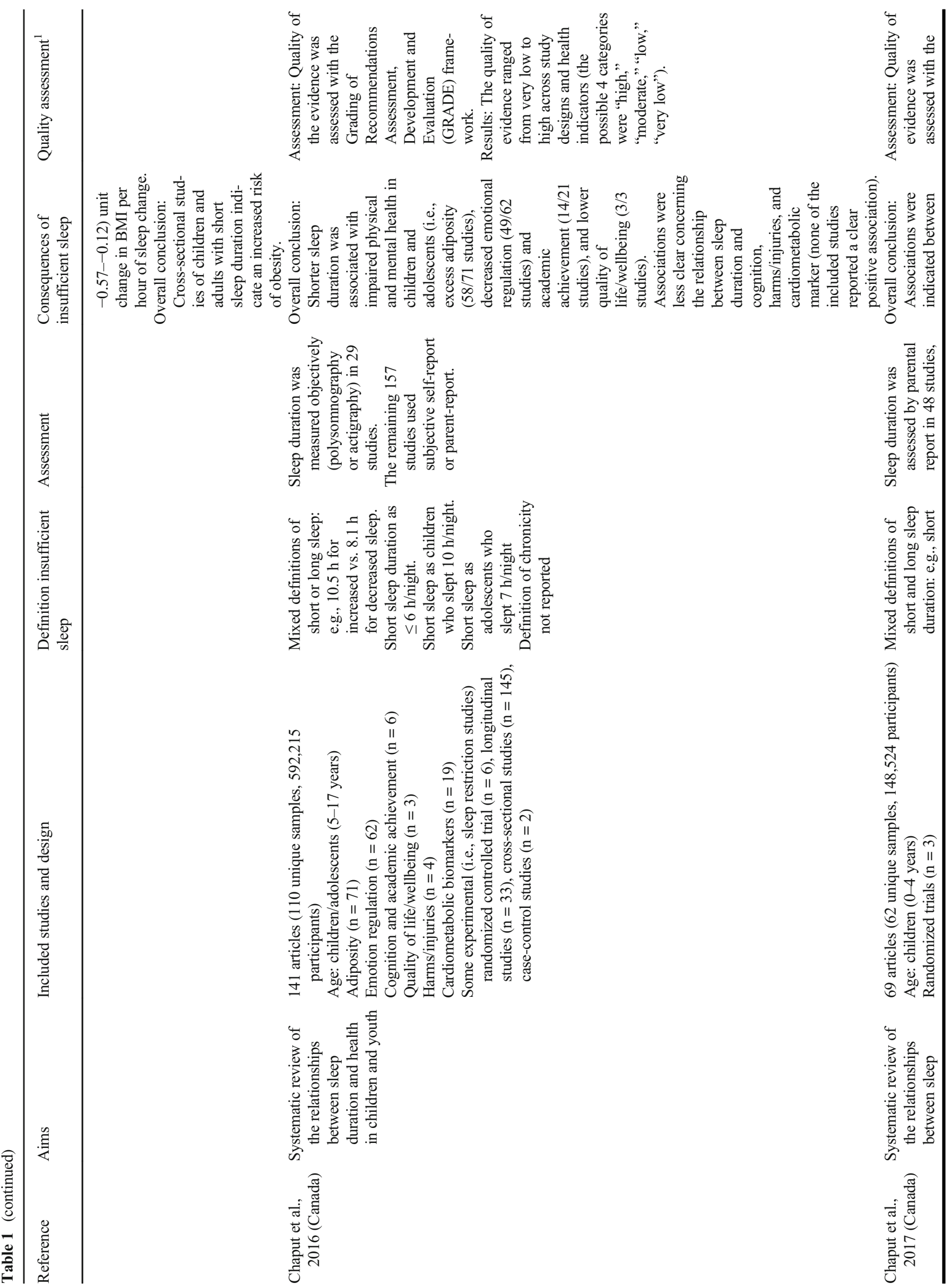




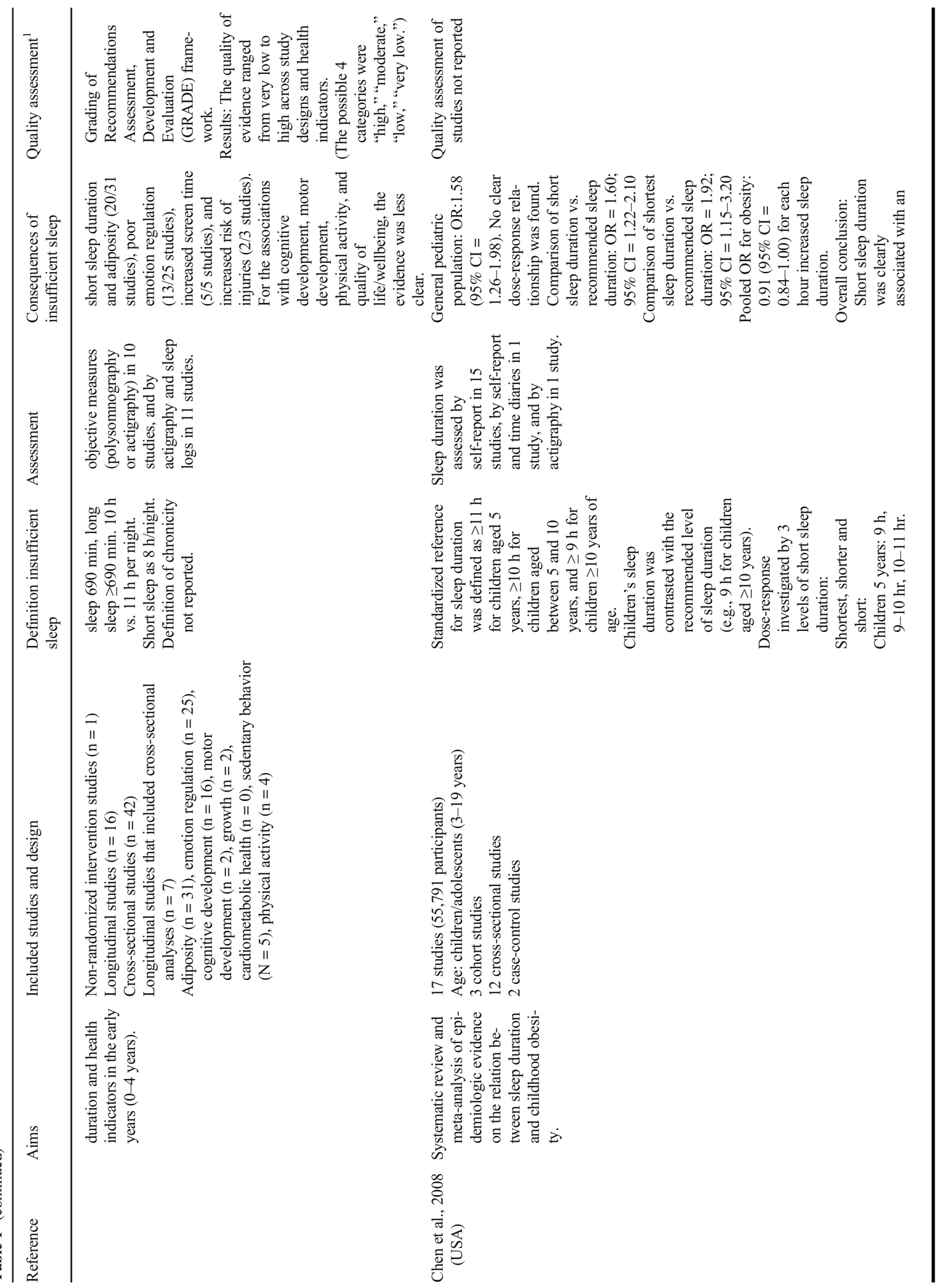




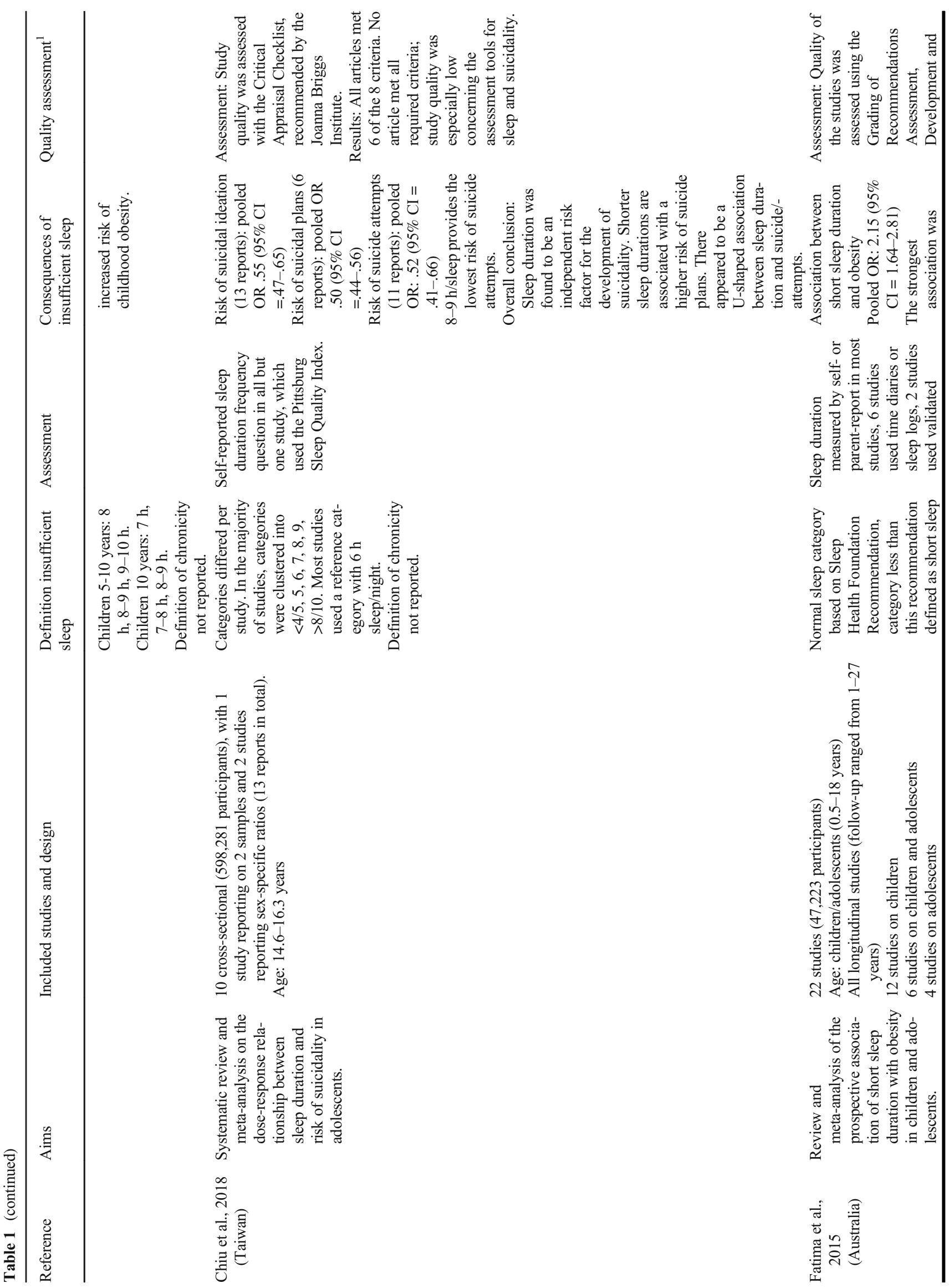




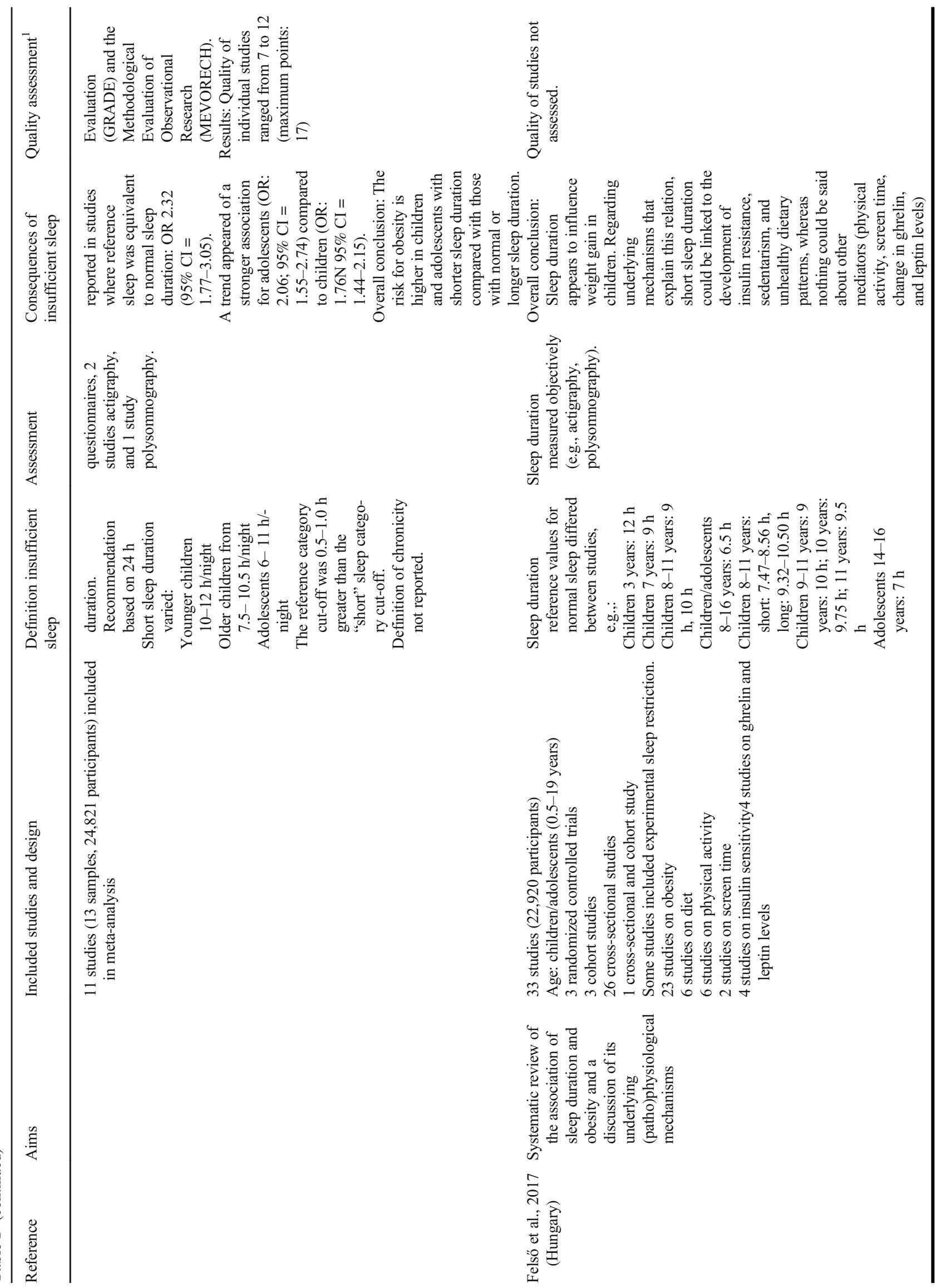




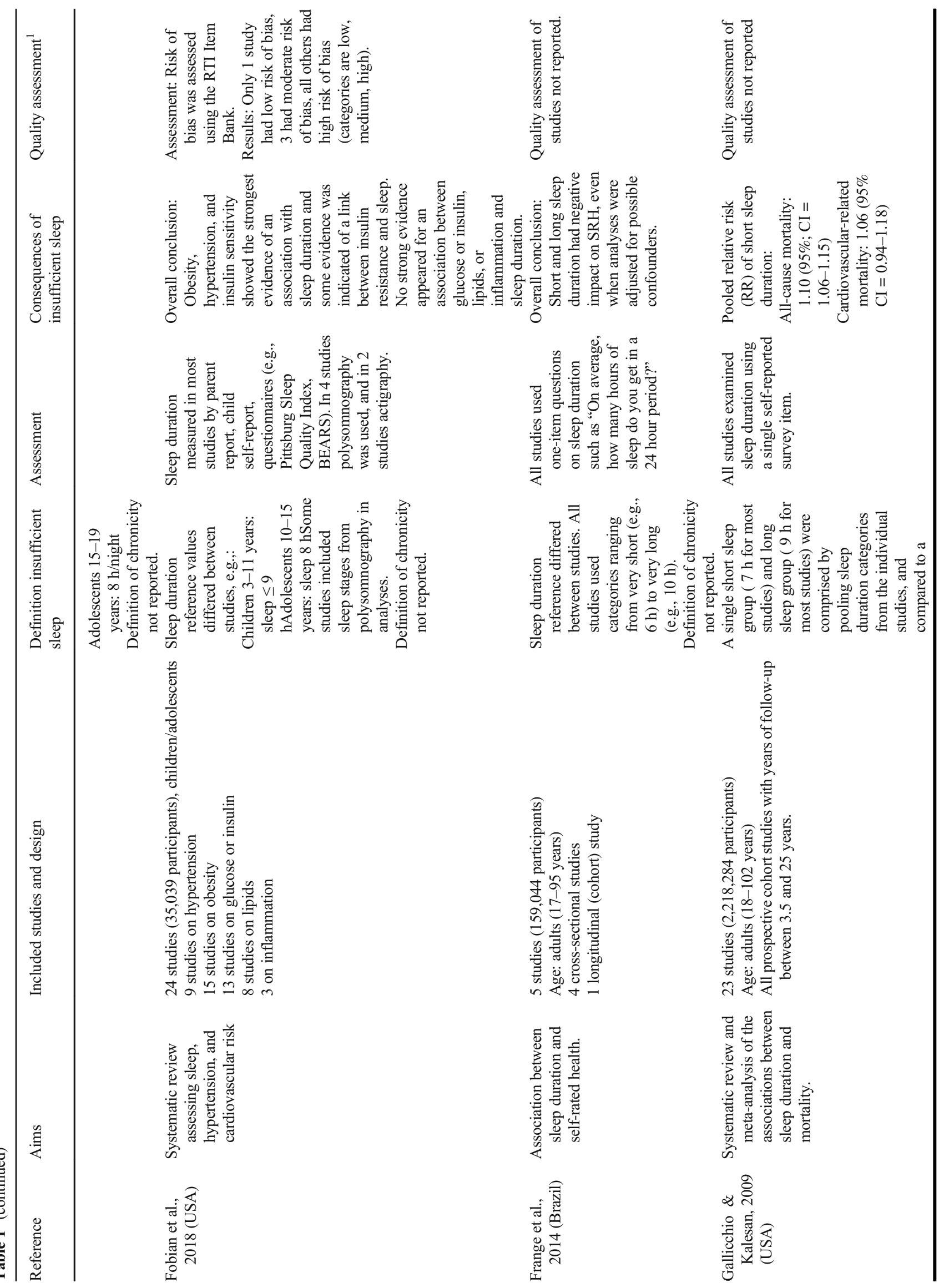




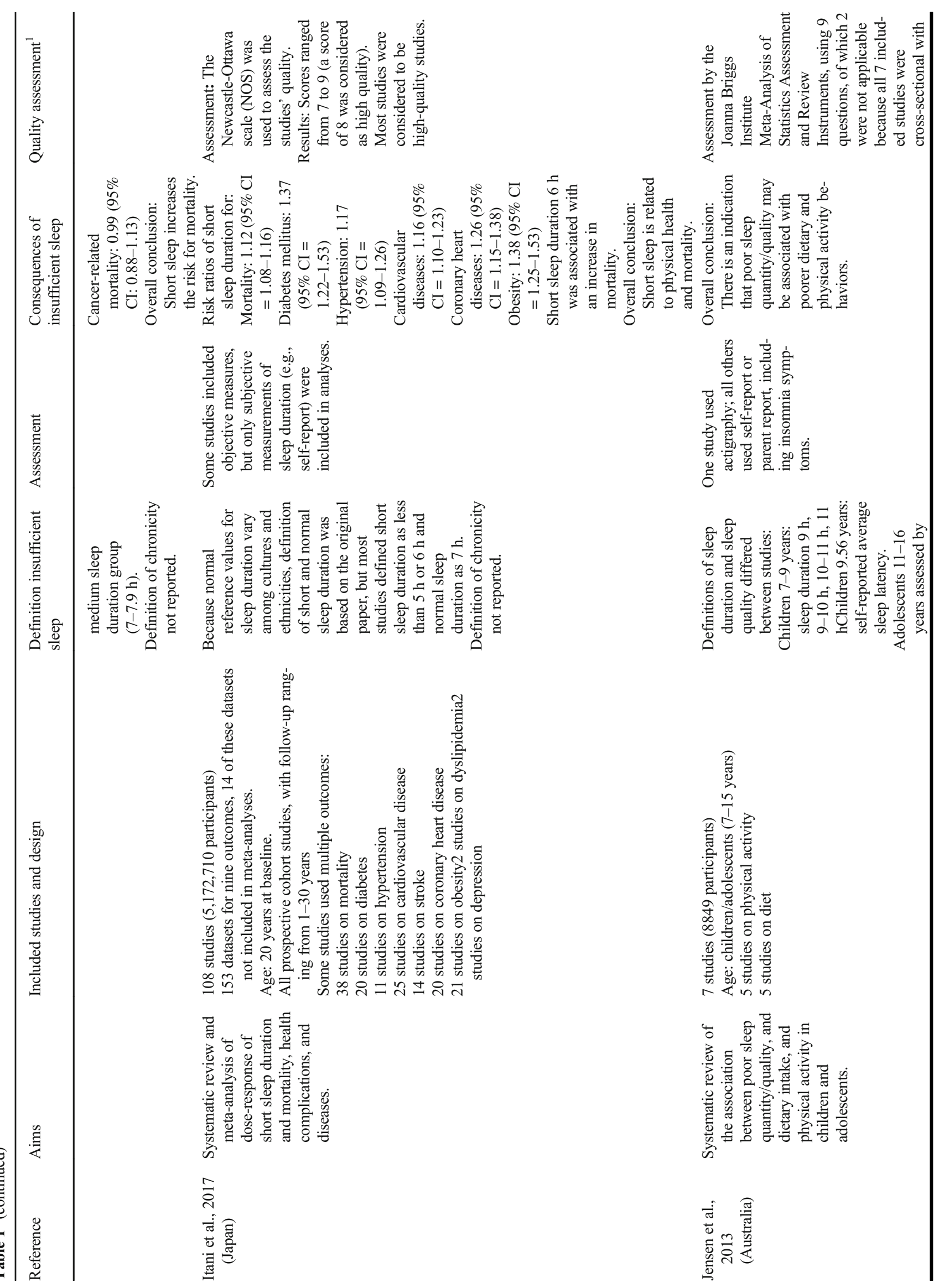




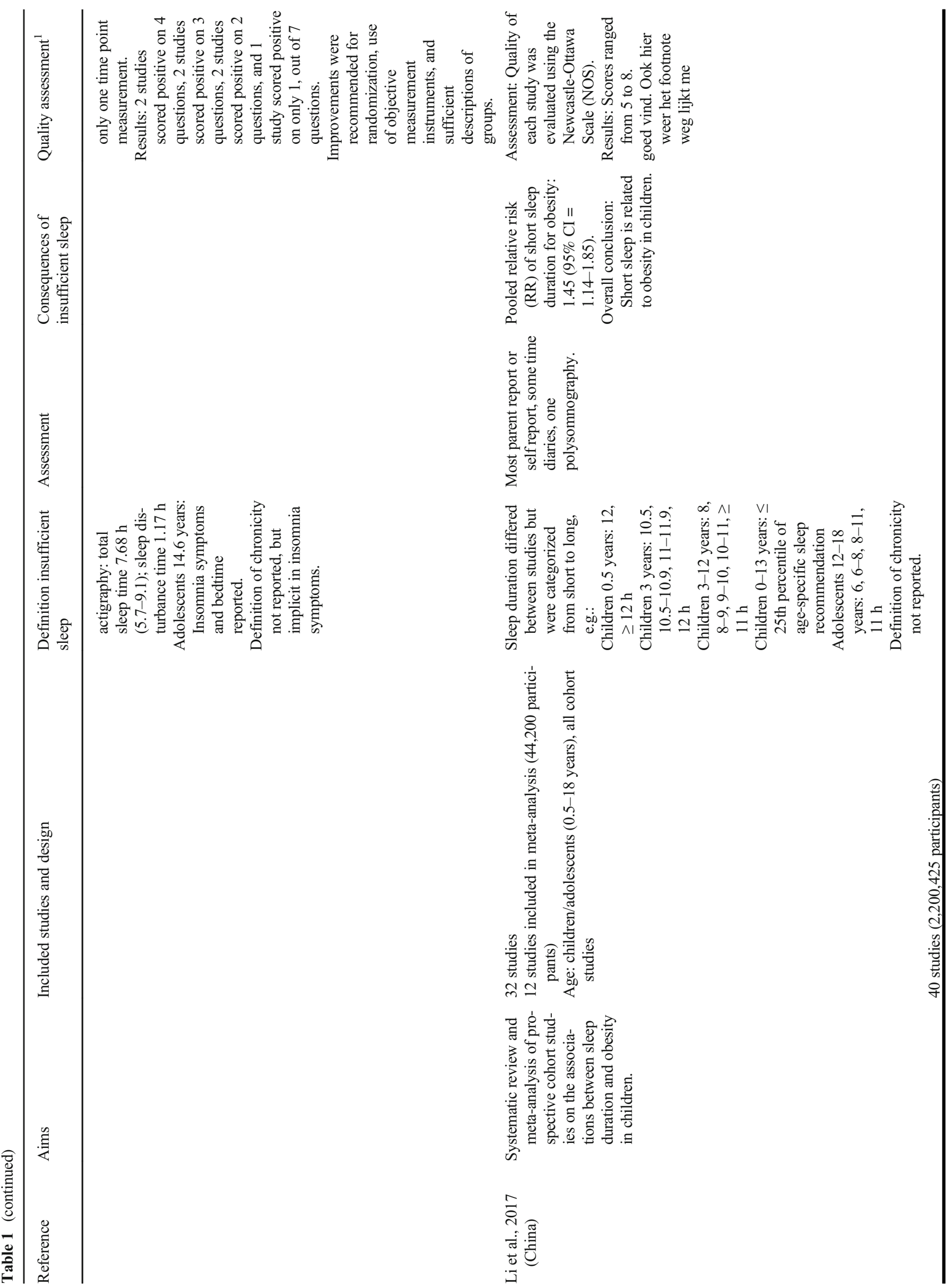




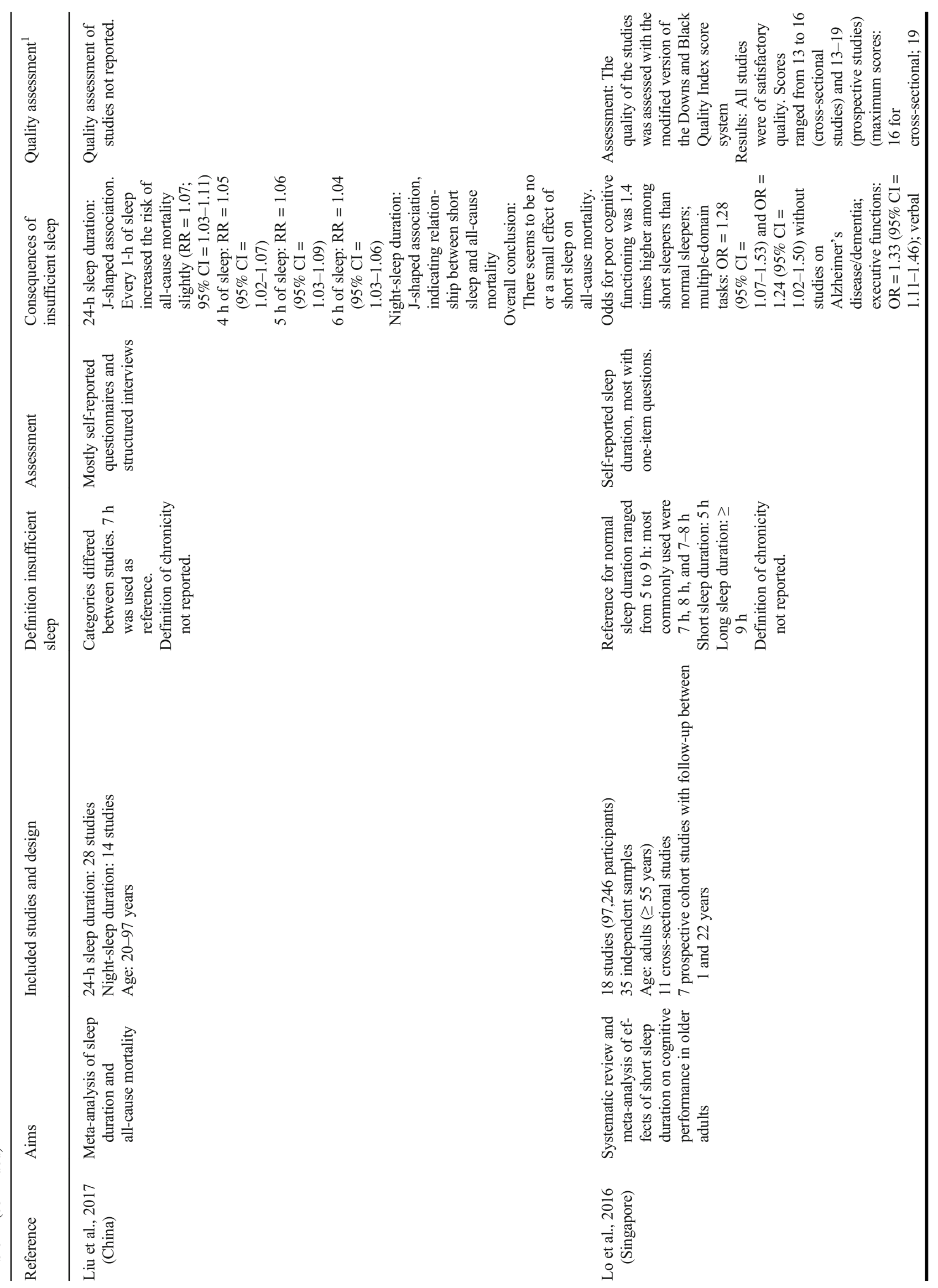




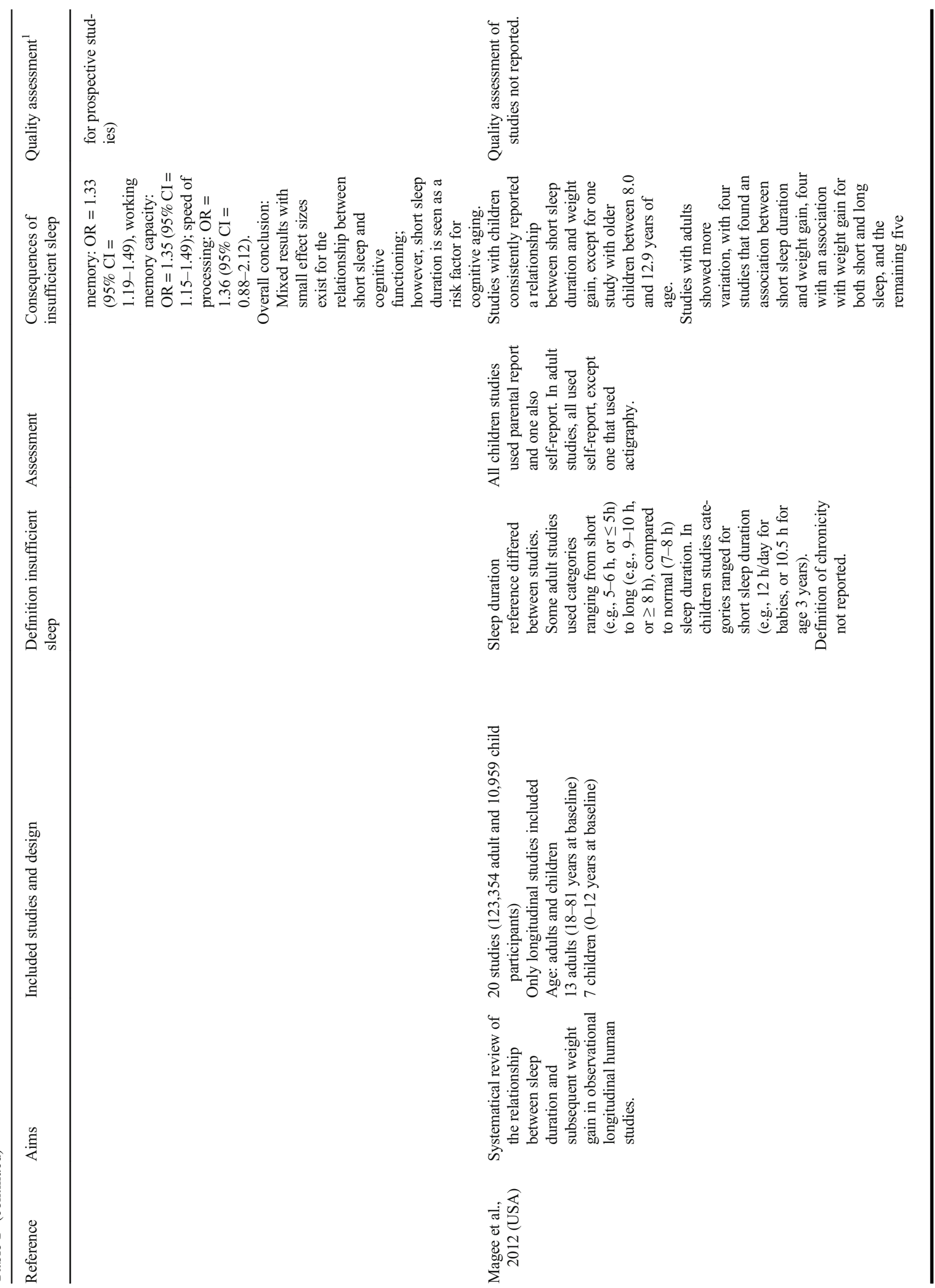




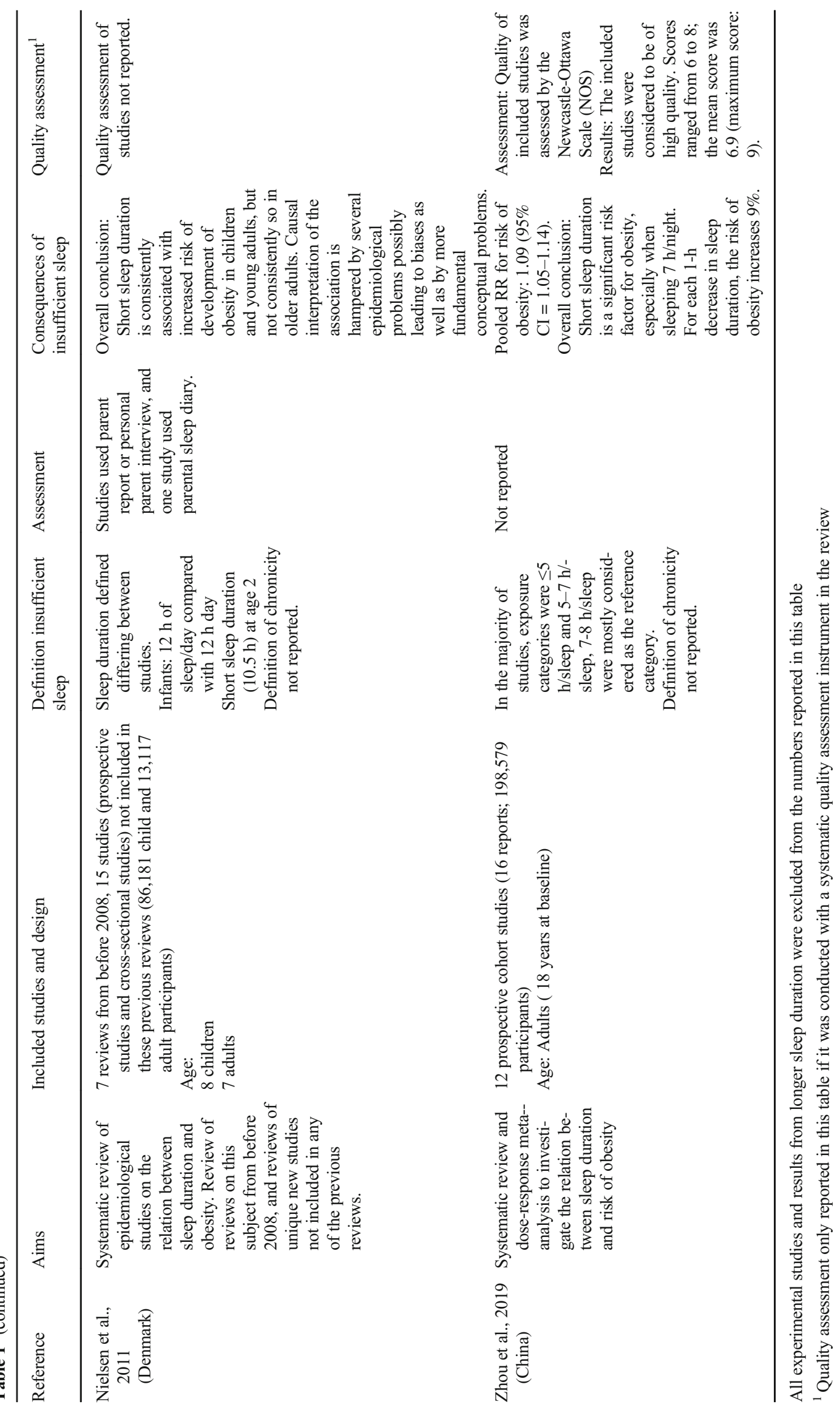




\section{Results}

Twenty-two articles $(13,504,230$ participants $)$ were included with 13 studies $(1,811,969$ participants) on children and/or adolescents and 12 studies $(11,692,261$ participants) on adults (three studies included both children/ adolescents and adults). The following domains of daytime functioning were affected by insufficient sleep: weight/obesity (13 studies), diabetes (four studies), cardiovascular diseases (five studies), cognitive functioning (four studies), emotional problems (three studies), mortality (three studies), and general health/quality of life (three studies). One study investigated the relationship between sleep duration and suicidality and a few reviews mentioned additional domains (e.g., physical activity, dietary, screen time), which could not be assigned to one of the above-mentioned domains (see Table 1). Overall, the results clearly show that the majority of reviews focused on physical health problems, with most articles addressing the relationship between insufficient sleep and obesity/weight, whereas only a limited number of reviews addressed the effects of insufficient sleep on behavior and cognitive functioning. The limited number of reviews on these topics may demonstrate a research gap of original studies addressing the effects of insufficient sleep on emotional and psychological wellbeing as well as on cognitive performance. Figure $2 \mathrm{a}-\mathrm{d}$ present the number of unique studies and participants divided into children/adolescents and adults for each domain separately.

\section{Chronic Sleep Reduction}

All included reviews provided data on sleep duration, meaning that studies gave a definition of short sleep by providing the exact hours of sleep. Although the definition of short sleep duration usually differed depending on the original studies rather than on theoretical grounds, in most reviews on adults, short sleep duration varied in the majority of included studies between 5 and $6 \mathrm{~h}$ of sleep per night [14-20]. Few reviews used slightly longer sleep durations (e.g., $7 \mathrm{~h}$ of sleep) as short sleep duration [17, 20-22, 23•]. The only article that additionally included other (actigraphic and polysomnographic) aspects of sleep quality was conducted by Aziz et al.[24], who defined sufficient sleep duration as $7 \mathrm{~h}$ sleep per night, but also used sleep efficiency $85 \%$, sleep fragmentation 25 , and proper sleep staging from polysomnography as indicators for sufficient sleep.

The definition of short sleep for children and adolescents differed from adults. Some reviews on children used sleep durations $9 / 10 \mathrm{~h}$ of sleep per night as an indication for insufficient sleep $[15,25]$, whereas others (additionally) specified sleep duration in more detail depending on different age categories $[25,26]$ or listed the different studies and their definitions of short sleep [27-32]. In most reviews, the definition depended on the original articles; however, Chen et al. [26] divided sleep duration into three categories (shortest, shorter, short). Fatima et al. [25] on the other hand created three groups with different definitions of insufficient sleep, which were based on the recommendations of the Sleep Health Foundation. Concerning adolescents, most studies used 6 to $8 \mathrm{~h}$ of sleep per night as insufficient sleep [25, 29, $32,33]$.

Although all included reviews aimed to assess the effects of insufficient sleep, operationalized by short sleep duration, on certain outcome variables, not one review provided a clear definition of chronicity, meaning that no review answered the question of how many nights sleep had to be insufficient or reduced. The reviews usually included studies that referred to an "average" or "general" sleep duration measured by a single question such as "how many hours of sleep do you usually get?" which indirectly includes chronicity as it implies that individuals receive that amount of sleep over a longer time period. Additionally, some reviews/meta-analyses reported that the measurement of usual sleep duration was indicated "over the last month" (e.g., [24]), for instance, when the Pittsburg Sleep Quality Index (PSQI; [34]) was used, or reported the use of sleep diaries, which typically measure one or 2 weeks of subjective sleep (e.g., [20]). Yet other studies reported the use of self-reported insomnia symptoms over a longer period (e.g., [31]). The only studies that defined some form of chronicity were the few experimental studies that were included in the reviews, such as $6.5 \mathrm{~h}$ in bed per night for 5 nights in one study included in the review by Chaput et al. [27]. However, this definition was related to experimental studies and is therefore excluded from this scoping review. Table 1 provides the detailed results of sleep duration for each review separately.

\section{Effects on Outcome Variables}

\section{Weight/Obesity}

We included five systematic reviews/meta-analyses on weight/obesity in adults (two systematic reviews, three studies combining meta-analysis and systematic review) that included 47 studies with 1,039,337 unique participants, and eleven systematic reviews/meta-analyses on children and adolescents (six systematic reviews, five studies combining metaanalysis and systematic review) that included 152 unique studies with 635,160 participants. Magee and Hale [19] pictured mixed results for the literature on adults when systematically reviewing longitudinal studies: Eight studies reported an association between short sleep duration and weight gain 

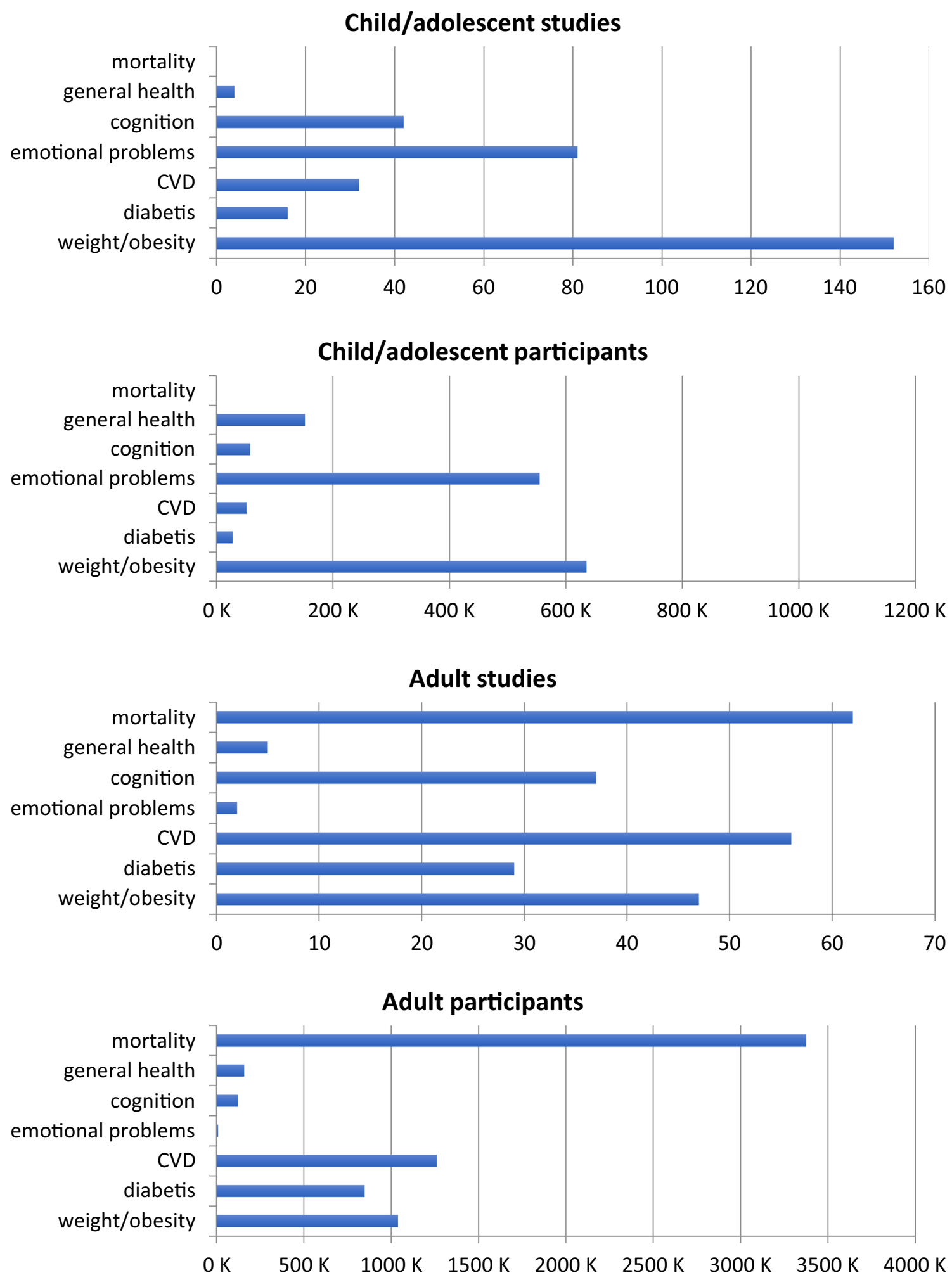

Fig. 2 a-d Numbers of unique child/adolescent and adult studies and participants included in the reviews and meta-analyses. NB. some outcome or mediator variables from the following articles could not be included in the categories of the graphs: adiposity, emotion regulation, growth, screen time, risk of injuries, cognitive development, motor development, physical activity, and quality of life/wellbeing [28]; suicidality [33]; insulin resistance, sedentarism, unhealthy dietary patterns, physical activity, screen time, change in ghrelin, and leptin levels [30]; inflammation [29]; sleep, diet, and physical activity [31] 
whereas five studies did not. Nielsen and colleagues [35] included both reviews of studies on adults and children from before 2008 as well as unique new studies published after 2008 and which were not part of the previous reviews. They concluded that in children and young adults, short sleep duration is consistently related to increased risk of obesity, but not in older adults. This finding supports the above-summarized evidence of adult studies. The meta-analysis of prospective cohort studies by Itani et al. [16] showed a clear effect of insufficient sleep on obesity as sleep duration $6 \mathrm{~h}$ per night was related to a significant increase in obesity. Comparable effects for cross-sectional studies were reported by Cappuccio et al. [15] and by Zhou et al. [23], who showed that the risk of obesity increases $9 \%$ for each 1-h decrease in sleep duration, when compared with $7 \mathrm{~h}$ of sleep per night.

Concerning children and adolescents, the systematic review on children by Fobian et al. [29] found the strongest evidence for an association between sleep and obesity, hypertension, and insulin sensitivity, and some evidence of a link between insulin resistance and sleep. There was no strong evidence of a relationship between glucose or insulin, lipids, or inflammation and sleep. Similarly, also Nielsen et al. [35], Felsö et al. [30], and Chaput et al. [27] found support for the idea that insufficient sleep influences weight gain and consequently the risk of obesity in children; however, the authors claim that the underlying explanatory mechanisms (e.g., physical activity) are still not fully understood. Still, it should be mentioned that not all included studies reported clear positive results. The conclusion for school-aged children and adolescents by Chaput et al. [27] was for instance based on 58 studies with significant associations and 13 studies reporting null findings. A similar effect was found in younger children aged 0-4 years [28], which was based on 20 studies reporting a positive association, nine studies with null findings, and two studies with an association between long sleep duration and adiposity. Based on the review of longitudinal studies, Magee and Hale [19] furthermore concluded that short sleep in children and adolescents is consistently related to weight gain. There was only one exception in one study that included older children (8.0-12.9 years at baseline and followed for 5 years). The meta-analytic evidence, which is based on cross-sectional, cohort, and case-control studies, by Chen et al. [26] showed that compared to children receiving sufficient sleep, children who have much shorter sleep duration also have an increased risk of obesity. Intriguingly, the group of children with the shortest sleep duration even had the highest risk of obesity. Similar results were found by Cappuccio et al. [15], who reported an increased risk of obesity for children with short sleep duration. Fatima et al. [25] showed in a meta-analysis of longitudinal studies that children and adolescents with short sleep double their risk to develop obesity compared with those who sleep longer with a stronger association for adolescents than for children. Miller et al. [36] showed in their meta- analysis on prospective studies that short sleep duration seems to be a risk factor or marker for the development of obesity in infants, children, and adolescents; however, studies per age group were rather small with three to eight studies per age group. Furthermore, the authors demonstrated that sleep duration was related to significant BMI changes. Li et al. [32] included only cohort studies in their meta-analysis and reported that short sleep duration increased the risk for obesity. To summarize, the existing systematic reviews and meta-analyses point towards an increased risk of weight gain/obesity in individuals with insufficient sleep.

\section{Diabetes}

We included two articles on diabetes combining a metaanalysis and systematic review in adults, which included 29 unique studies with 848,011 participants, and two systematic reviews in children and adolescents, which included 16 unique studies with 27,804 participants. The systematic review and meta-analysis on cohort studies of adults by Anothaisintawee et al. [14] reported a significantly small effect of sleep for diabetes. Comparable results were reported by Itani et al. [16], who found that a sleep duration $6 \mathrm{~h}$ per night was associated with a significant increase in diabetes.

No study directly assessed the association between sleep and diabetes in children and adolescents; however, Felsö et al. [30] verified in their systematic review of studies with mixed study designs that short sleep duration is related to the development of insulin resistance, sedentarism, and unhealthy dietary patterns. Similarly, Fobian et al. [29] reported a strong relationship between sleep and obesity, hypertension, and insulin sensitivity, and some evidence of a link between insulin resistance and sleep. There was no strong evidence of a relationship between glucose or insulin, lipids, or inflammation and sleep. It can therefore be concluded that short sleep seems to increase the risk to develop diabetes or health conditions that are related to diabetes, in all age groups.

\section{Cardiovascular Disease}

We included two articles on cardiovascular disease in adults (one systematic review, one study including a systematic review and meta-analysis), which included 56 unique studies with $1,260,193$ participants, and three systematic reviews in children and adolescents, which included 32 unique studies with 51,855 participants. Aziz et al. [24] conclude that in cohort studies with adults, a relationship of sleep with increased subclinical cardiovascular disease burden; however, it has to be considered that mixed results were reported by the included studies. Itani et al. [16] were the only authors who conducted a meta-analysis and indicated a threshold of sleep duration, by stating that a sleep duration $6 \mathrm{~h}$ per night was associated with a significant increase in cardiovascular 
diseases. Mixed findings for sleep and cardiometabolic markers in children and adolescents aged $5-17$ years were shown by Chaput et al. [27] as some studies being included in their review found a positive association whereas others reported null findings. In another review from Chaput et al. [28] on children aged $0-4$ years, the authors found no studies that investigated the association between sleep duration and cardiometabolic biomarkers. Fobian et al. [29] found in their systematic review on children, in which mixed study designs were included, the strongest evidence of an association with sleep for obesity, hypertension, and insulin sensitivity, and some evidence of a link between insulin resistance and sleep, whereas there was no strong evidence of a relationship between glucose or insulin, lipids, or inflammation and sleep.

In summary, although the existing reviews suggest a relationship between short sleep duration and cardiovascular disease, it seems to be rather small and complex. More research focusing on this complexity should therefore be conducted and compared in systematic reviews and/or meta-analyses.

\section{Cognitive Functioning}

We included two articles on cognitive functioning in adults (one systematic review and one study including a systematic review and meta-analysis), which included 37 unique studies with 124,696 participants, and two systematic reviews in children and adolescents, which included 42 unique studies on cognitive functioning with 57,993 participants. Brewster et al. [20] included cross-sectional, prospective, and longitudinal studies in their systematic review and found mixed results with indications of a U-shaped relationship between sleep and cognitive functioning in older adults. The metaanalysis by Lo et al. [18], which included cross-sectional and prospective cohort studies, found a small effects size for the relationship between sleep duration and cognitive functioning. The effect differed depending on cognitive domains, as insufficient sleep was significantly associated with poorer performance in multiple-domain tasks, executive functioning, verbal memory, and memory capacity but not with speed of processing. Chaput et al. [27] included studies on children and adolescents and Chaput et al. [28] even investigated the relationship between sleep duration and cognitive development in children aged 0 to 4 years. Both reviews, including mixed study designs, found mixed results for the association between sleep duration and cognition, which is in line with the reviews on adults. Academic achievement, which is closely related to cognitive functioning, on the other hand, was found to be critically related to shortened sleep [27]. Interestingly, none of the reviews included a clear cut-off indicating sufficient sleep, which would allow drawing conclusions on the amount and period of insufficient sleep needed in order to significantly affect cognitive functioning. It can therefore be concluded that the relationship between insufficient sleep and cognitive performance seems to be small and complex as the results of the only meta-analysis highlight the need to carefully differentiate between different cognitive domains.

\section{Emotional Problems}

We included one meta-analysis on emotional problems in adults that included two unique studies with 10,096 participants, and two systematic reviews in children and adolescents that included unique 81 studies with 554,978 participants. The study by Itani et al. [16] was the only meta-analysis on studies with adults that aimed to include depression as the outcome variable. However, the authors were unable to conduct the analysis as the amount of available evidence was too small. Therefore, no conclusion about the association between sleep and emotional problems in adults can be drawn. Based on 49 studies reporting that long sleep duration was associated with better emotion regulation, eleven studies with null findings and two studies with opposite findings, Chaput et al. [27] conclude that insufficient sleep is related to more emotion regulation problems in children and adolescents aged 5-17 years. A similar relationship was found in a review on younger children aged 0 to 4 years, which was based on 13 studies reporting a significant effect, ten studies reporting null findings, and two studies that found an association between longer sleep duration and poorer emotional regulation [28]. It remains an interesting research gap that no results from a systematic review or meta-analysis in adults were available and that the empirical evidence in children and adolescents is rather limited.

\section{General Health/Quality of Life}

We included one systematic review on general health in adults that included five studies with 159,044 participants, and two systematic reviews on children and adolescents that included four studies with 151,834 participants. Concerning general health in adults, Frange et al. [17] found in their systematic review on mainly cross-sectional studies that sleep duration negatively affects self-reported health even when analyses were adjusted for possible confounders. However, as mentioned above, this systematic review was based on only five studies and should therefore be carefully interpreted.

Chaput et al. [27] conclude in their review on school-aged children and adolescents that an association between sleep duration and quality of life exists, whereas the evidence for the relationship between sleep duration and harm/injury was mixed. However, it has to be mentioned that the conclusion on quality of life could only be based on three studies and that all reported an effect and the conclusion on harm/injury was based on four studies, with one study reporting a positive effect, one study reporting no effect, and two studies reporting mixed results. In younger children, aged 0 to 4 years, the 
review by Chaput et al. [28] found that growth, risk of injuries, and screen time are affected by shortened sleep, whereas results seem to be less clear for motor development, physical activity, and quality of life. This may at least partially be caused by the very limited number of studies on these topics, which makes it difficult to compare study results.

These results show that little research has been done investigating the relationship between insufficient sleep and general health, both in adults and children. This includes the number of studies that could be included in the reviews as well as the number of existing reviews with not one meta-analysis.

\section{Mortality}

We included three meta-analyses on the association between sleep and mortality in adults that included 62 studies with $3,374,285$ unique participants. No systematic review/metaanalysis on children or adolescents was found. Based on the review of prospective cohort studies by Gallicchio and Kalesan [21], it can be concluded that short sleepers are at higher risk of mortality. Similar results from prospective cohort studies were reported by Itani et al. [16], who even report that a sleep duration $6 \mathrm{~h} /$ night was associated with a significant increase in mortality (as well as in diabetes, cardiovascular disease, and obesity). Liu et al. [22] found in their metaanalysis on prospective cohort studies a J-shaped association between sleep duration over $24 \mathrm{~h}$ (including daytime sleep) and all-cause mortality. They reported that every hour of reduced sleep increased the risk of all-cause mortality. However, concerning night-sleep duration only, less than $7 \mathrm{~h}$ did not increase the risk of all-cause mortality.

\section{Study Quality}

Out of the 22 included reviews/meta-analyses, only 12 reported on the quality of the included studies (see Table 1 for a detailed overview). The results were heterogeneous, ranging from studies with very low to high quality. However, reviews used different assessment tools, to measure study quality. As these tools also focused on different aspects of study quality (e.g., study design, validity and reliability of measurements, follow-up measures), the reported results are difficult to compare. Still, we highly recommend including quality assessments in reviews/meta-analyses, as it helps to interpret the described results and conclusions.

\section{Subgroup Analyses and Health Disparities}

Literature indicates that socio-demographic variables, such as age, gender, income, educational level, and race/ethnicity, can influence the duration and quality of sleep. In order to assess health disparities, a meta-analysis rather than a systematic review is needed as this would allow an estimation of the exact effect for subgroups. In response to this, many of the included meta-analyses used these variables to control for possible confounding effect and to identify the pure effect of sleep duration and quality on health outcomes. In some cases, this led to including subgroup analyses based on age (e.g., $[15,16,18$, $22,23,25,26,32,36])$ and/or gender (e.g., $[16,18,21-23$, $26]$ ), for which results have been described above. However, from the included meta-analyses, it is not possible to draw direct conclusions on health disparities between populations with different socio-demographic characteristics, such as race/ethnicity, income, and educational level, as health disparities were not the primary aim of the meta-analyses. It also has to be mentioned that studies often controlled for influential factors, meaning that the meta-analyses were using effects that were already controlled for possible confounding variables in the original studies.

\section{Discussion}

The results from our scoping review regarding the aim to provide an overview of chronic sleep reduction show that a clear definition of chronic sleep reduction is lacking. None of the included reviews/meta-analyses explicitly addressed the question of chronicity. Studies usually reported an average sleep duration or defined insufficient sleep on average (e.g., $7 \mathrm{~h}$ per night) and measured sleep duration with one single question ("e.g., how many hours do you usually sleep at night?"). Regarding the second and third aims of this scoping review, namely to summarize the effects of short sleep on daytime functioning and identify research gaps, the results showed that short sleep duration is related to obesity and diabetes. Relations were also demonstrated with CVD, cognitive functioning, emotional problems, general health, and mortality, but the effects were mostly small, the relations appeared complex (e.g., for CVD and cognitive functioning), and the numbers of studies were small or completely lacking (e.g., mortality in children/adolescents, and emotional problems in adults).

Although the lack of clearly defined criteria for chronicity in all studies seems surprising at first sight, the following research challenges may help to explain this result: most studies that were included in the reviews/meta-analyses had crosssectional designs, in which participants were simply asked to indicate their average sleep duration. More complex or objective aspects of sleep were not included, and therefore, the systematic reviews or meta-analyses were unable to address the question of chronicity (e.g., time period during which insufficient sleep has to occur to affect functioning) or even the direction of the relations between sleep and other outcomes. Furthermore, it is striking that in many instances the original studies that were included in these reviews/meta-analyses did not report criteria for chronicity either. Considering the fact 
that in experimental studies a dose-response relationship between sleep and performance has been demonstrated (e.g., [37]), this lack of definitions and reporting of the time period of sleep duration is an important omission in the current literature. In some reviews/meta-analyses, studies were included that measured sleep with sleep logs and/or actigraphy over 7 days, or used polysomnography. These measures of sleep duration may be more reliable than a single self-report item in a long questionnaire, but it is unclear how these measures relate to chronicity, meaning how sleep duration from for example polysomnography is related to usual sleep duration over a longer time period. Another important issue refers to the phenomenon of individual sleep need, which can be defined as the amount of sleep somebody needs without being influenced by previous sleep debt [38]. Individual sleep need was shown to range from 7.5 to $8.5 \mathrm{~h}$ /sleep per night [39] with an average sleep need of 8.16 [37] for adults, and $9.35 \mathrm{~h}$ for adolescents [40•], and has a genetic component with $31 \%$ to $55 \%$ heritability [41-43]. From this existing evidence, it can be concluded that the effects of sleep duration should be interpreted with reference to ones' individual sleep need; however, this facet of sleep was not addressed in the included reviews/metaanalyses.

One attempt to overcome the problem of the influence of individual sleep need on optimal sleep duration has been provided by Meijer [44], who developed the "Chronic Sleep Reduction Questionnaire (CSRQ)" for adolescents [44, 45]. This questionnaire measures daytime problems rather than sleep directly and assumes that adolescents only score high on these problems if they experience less sleep than needed over a prolonged time period. Although this seems to be an interesting approach, also this questionnaire does not give an evidence-based rationale for the time period of 2 weeks, during which these daytime symptoms have to occur. In contrast, the DSM-5 defines that symptoms of insomnia must be present for at least 3 months to fulfill the criteria of the diagnosis "chronic insomnia"[5] similar to the ICSD-3 classification of "Chronic Insomnia Disorder" [46]. However, the DSM-5 also claims that individuals experience impairments in their daytime functioning [5], indirectly indicating that individuals sleep less than their individual sleep need. Although this idea is in line with the assumption of the CSRQ, based on the results of this scoping review, it remains unclear whether the CSRQ subscales (shortness of sleep, sleepiness, irritation, loss of energy) represent the best empirically supported categories. We found that especially physical health was related to insufficient sleep, whereas the evidence for the relationship between sleep and emotional or psychological wellbeing was too limited to draw clear conclusions (see discussion below). Furthermore, we did not find reviews on the effects of insufficient sleep on daytime sleepiness, one of the CSRQ subscales.
Concerning the outcomes of daytime functioning related to short sleep duration, the results of this scoping review show that most reviews addressed weight gain/obesity both in children/adolescents and in adults, and also found the most robust association, indicating that insufficient sleep is related to weight gain and/or even obesity. Some reviews attempted to identify possible mediators (e.g., dietary, physical activity, screen time) that may play a role within this association. Although no strong evidence of these factors could be confirmed by the reviews so far, the existing empirical evidence is limited and future studies should further investigate their role in order to understand the complexity of the effects of insufficient sleep. The effects on diabetes and CVD seem to be less stable, as especially mixed results were found for the relationship between sleep and the latter. Mortality and general health seem to be related to sleep reduction; however, again the available empirical evidence is rather limited and reviews were often based on the results of only a few studies. Moreover, the largest body of literature consists of large cross-sectional studies that is limited for causal inference, and mostly used single-item self or parent report of sleep duration. The limitations of these methods have been outlined above, and results should be interpreted with caution regarding direction of the risk, and validity of the measurements.

Although the literature often postulates that sleep affects cognitive functioning [47-49], our review points towards mixed results and rather small effect sizes. This may especially be caused by the finding that sleep seems to affect certain aspects of cognitive functioning (e.g., multiple-domain tasks, executive functioning, verbal memory, memory capacity), which refer to higher order cognitive functioning, whereas more basic cognitive aspects (e.g., speed of processing) seem to be unaffected. This finding is in line with other reviews that found sleep to be related to some, but not all areas of cognitive functioning [50, 51] (these reviews were not included in this scoping review as they did not meet the inclusion criteria). Reynoud et al. [50] furthermore found more significant effects for studies with larger sample sizes, which supports the idea that effect sizes seem to be rather small and large sample sizes are needed to detect effects and to ensure sufficient statistical power. Interestingly, also academic achievement, which requires cognitive functioning, seems to be related to shortened sleep. This finding was also supported by two meta-analyses in children and adolescents [52, 53]; however, it has to be mentioned that also these meta-analyses reported small effect sizes, indicating that additional factors seem to play an important role. We highly recommend focusing future research on such mediators within the long-term relationship between sleep and cognitive functioning and/or academic achievement. Surprisingly little evidence was found for the relationship between sleep and emotional problems, although experimental studies and clinical groups point towards an interplay between sleep problems, mood, and psychiatric problems [54]. It was 
even shown that sleep problems are already present prior to the development or recurrence of depression [55] and Beatti et al. [56] conclude in their systematic review that sleep deprivation affects emotion recognition, measured with experimental paradigms. These effects were not only seen on a behavioral or emotional level but also affected brain activity [56], pointing towards underlying neural correlates. The present scoping review therefore identifies an important research gap in the area of sleep and emotional problems in healthy samples, which should be addressed in future studies, optimally in (prospective) cohort or longitudinal study designs.

Overall, the study quality of included studies was rather low for all outcome variables. Therefore, more high-quality research should be conducted in the future in order to draw valid conclusions about the effects of insufficient sleep on daytime functioning. The finding that the majority of reviews/meta-analysis investigated weight gain/obesity, whereas cognitive functioning, for example, appeared in much fewer numbers, especially if compared in numbers of participants that were included in the studies, could be partially due to practical and financial constraints that are inherent to measuring cognitive functioning in large cohorts. However, the inherent complexity of measuring cognitive functioning could be argued to be present as well in measuring sleep. As discussed above, sleep duration in most reviews/metaanalyses was measured with single-item questions, which disregards chronicity, quality, variability, fragmentation, timing, and individual sleep need. Moreover, measuring weight gain/obesity requires more than a single item on a questionnaire and can be regarded as a somewhat labor-intensive measure. It seems that especially in larger cohort studies that adopt such single-item questions on sleep duration, this complexity has been underestimated. It is therefore highly recommended to adjust the method of sleep measurement in epidemiological studies, which is in concurrence with previous recommendations $[57,58]$.

\section{Limitations}

The following limitations should be mentioned: (1) there is a big heterogeneity not only between original studies but also between the included systematic reviews and meta-analyses, which makes it difficult to directly compare the results; (2) many studies measure sleep duration with one single item, which may lead to biased results; (3) the study quality of most studies was rather low; (4) as we included only previous systematic reviews or meta-analyses rather than original studies, lacking evidence on some outcome domains (e.g., behavioral problems, emotional problems) may either result from a small number of original studies, representing a research gap in this area, or from a lack of reviews on this topic; (5) the results clearly demonstrate a lack of definitions for chronicity of insufficient sleep and it was therefore impossible to provide a definition of chronic sleep reduction, which (as stated in the introduction) is highly needed for future research and clinical practice.

\section{Future Directions and Research Agenda}

We highly recommend more studies that aim to define the chronicity of insufficient sleep. This first attempt should be to experimentally clarify the dose-response relationship between insufficient sleep and outcome variables, followed by more naturalistic, quasi-experimental studies in individuals' daily life and natural environment. Additionally, influential factors, including mediating, and moderating effects, and health disparities between different socio-demographic populations, should be assessed. We also recommend focusing more on resilience and protective factors that may buffer the negative effects of insufficient sleep. More knowledge on these topics is highly relevant for future prevention and intervention studies that aim to improve daytime functioning and physical and/or psychological health by improving individuals' sleep. Regarding outcomes, we recommend further research on areas that have been demonstrated to be affected by sleep reduction in experimental research, but are largely lacking in cohort studies, such as emotional and behavioral problems, and cognitive functioning. Furthermore, research should focus to develop more specific and clear evidence-based guidelines on chronic sleep reduction and its effects, and develop validated measures that can replace the existing methods in larger epidemiological or cohort studies. Such guidelines would be helpful for future research, as they would help to make studies more comparable and guide clinical practitioners.

Supplementary Information The online version contains supplementary material available at https://doi.org/10.1007/s40675-021-00214-1.

Acknowledgements The authors wish to thank Janneke Staaks for her assistance in the systematic search.

Code Availability Not applicable

Author Contribution Both authors contributed equally to the manuscript and study.

Availability of Data and Material Not applicable

\section{Compliance with Ethical Standards}

Conflict of Interest The authors declare that they have no conflict of interest.

Human and Animal Rights and Informed Consent Not applicable. 
Consent for Publication The authors agree to the terms and conditions for publication as stated by the publisher of the journal.

Open Access This article is licensed under a Creative Commons Attribution 4.0 International License, which permits use, sharing, adaptation, distribution and reproduction in any medium or format, as long as you give appropriate credit to the original author(s) and the source, provide a link to the Creative Commons licence, and indicate if changes were made. The images or other third party material in this article are included in the article's Creative Commons licence, unless indicated otherwise in a credit line to the material. If material is not included in the article's Creative Commons licence and your intended use is not permitted by statutory regulation or exceeds the permitted use, you will need to obtain permission directly from the copyright holder. To view a copy of this licence, visit http://creativecommons.org/licenses/by/4.0/.

\section{References}

Papers of particular interest, published recently, have been highlighted as:

- Of importance

1. Meltzer LJ. Future directions in sleep and developmental psychopathology. J Clin Child Adolesc Psychol. 2017;46(2):295-301. https://doi.org/10.1080/15374416.2016.1236727.

2. Eidelman P, Gershon A, McGlinchey E, Harvey A. Sleep and psychopathology. In: Espie CA, Morin CM, editors. The Oxford handbook of sleep and sleep disorders. Oxford: Oxford University Press; 2012.

3. Grandner MA, Hale L, Moore M, Patel NP. Mortality associated with short sleep duration: the evidence, the possible mechanisms, and the future. Sleep Med Rev. 2010;14(3):191-203. https://doi. org/10.1016/j.smrv.2009.07.006.

4. Szentkiralyi A, Madarasz CZ, Novak M. Sleep disorders: impact on daytime functioning and quality of life. Expert Rev Pharmacoecon Outcomes Res. 2009;9(1):49-64. https://doi.org/10.1586/ 14737167.9.1.49.

5. Association AP. Diagnostic and statistical manual of mental disorders: DSM-5. 5th ed. Arlington, VA: American Psychiatric Association; 2013.

6. Grandner MA, Patel NP, Gehrman PR, Perlis ML, Pack AI. Problems associated with short sleep: bridging the gap between laboratory and epidemiological studies. Sleep Med Rev. 2010;14(4):239-47. https://doi.org/10.1016/j.smrv.2009.08.001.

7. Ford ES, Cunningham TJ, Croft JB. Trends in self-reported sleep duration among US adults from 1985 to 2012. Sleep. 2015;38(5): 829-32. https://doi.org/10.5665/sleep.4684.

8. Keyes KM, Maslowsky J, Hamilton A, Schulenberg J. The great sleep recession: changes in sleep duration among US adolescents, 1991-2012. Pediatrics. 2015;135(3):460-8. https://doi.org/10. 1542/peds.2014-2707.

9. Watson NF, Badr MS, Belenky G, Bliwise DL, Buxton OM, Buysse D, et al. Recommended amount of sleep for a healthy adult: a joint consensus statement of the American Academy of Sleep Medicine and Sleep Research Society. Sleep. 2015;38(6):843-4. https://doi.org/10.5665/sleep.4716.

10. Buysse DJ. Sleep health: can we define it? Does it matter? Sleep. 2014;37(1):9-17. https://doi.org/10.5665/sleep.3298.

11. Armstrong R, Hall BJ, Doyle J, Waters E. Cochrane Update. 'Scoping the scope' of a cochrane review. J Public Health. 2011;33(1):147-50. https://doi.org/10.1093/pubmed/fdr015.
12. Matricciani L, Paquet C, Galland B, Short M, Olds T. Children's sleep and health: a meta-review. Sleep Med Rev. 2019;46:136-50. https://doi.org/10.1016/j.smrv.2019.04.011 A clear summary of the current knowledge concerning the relationship between sleep, developmental aspects, and health in children and adolescents, which is complementary to the results in our current scoping review.

13. Spruyt K. A review of developmental consequences of poor sleep in childhood. Sleep Med. 2019;60:3-12. https://doi.org/10.1016/j. sleep.2018.11.021.

14. Anothaisintawee T, Reutrakul S, Van Cauter E, Thakkinstian A. Sleep disturbances compared to traditional risk factors for diabetes development: systematic review and meta-analysis. Sleep Med Rev. 2016;30:11-24. https://doi.org/10.1016/j.smrv.2015.10.002.

15. Cappuccio FP, Taggart FM, Kandala N-B, Currie A, Peile E, Stranges S, et al. Meta-analysis of short sleep duration and obesity in children and adults. Sleep. 2008;31(5):619-26.

16. Itani O, Jike M, Watanabe N, Kaneita Y. Short sleep duration and health outcomes: a systematic review, meta-analysis, and meta-regression. Sleep Med. 2017;32:246-56. https://doi.org/10.1016/j. sleep.2016.08.006.

17. Frange C, de Queiroz SS, da Silva Prado JM, Tufik S, de Mello MT. The impact of sleep duration on self-rated health. Sleep Sci. 2014;7(2):107-13. https://doi.org/10.1016/j.slsci.2014.09.006.

18. Lo JC, Groeger JA, Cheng GH, Dijk D-J, Chee MWL. Selfreported sleep duration and cognitive performance in older adults: a systematic review and meta-analysis. Sleep Med. 2016;17:87-98.

19. Magee L, Hale L. Longitudinal associations between sleep duration and subsequent weight gain: a systematic review. Sleep Med Rev. 2012;16(3):231-41.

20. Brewster GS, Varrasse M, Rowe M. Sleep and cognition in community-dwelling older adults: a review of literature. Healthcare (Basel, Switzerland). 2015;3(4):1243-70.

21. Gallicchio L, Kalesan B. Sleep duration and mortality: a systematic review and meta-analysis. J Sleep Res. 2009;18(2):148-58. https:// doi.org/10.1111/j.1365-2869.2008.00732.x.

22. Liu TZ, Xu C, Rota M, Cai H, Zhang C, Shi MJ, et al. Sleep duration and risk of all-cause mortality: a flexible, non-linear, meta-regression of 40 prospective cohort studies. Sleep Med Rev. 2017;32:28-36. https://doi.org/10.1016/j.smrv.2016.02.005.

23. Zhou Q, Zhang M, Hu D. Dose-response association between sleep duration and obesity risk: a systematic review and meta-analysis of prospective cohort studies. Sleep Breath. 2019;23(4):1035-45. https://doi.org/10.1007/s1132 A comprehensive review and meta-analysis of the evidence of dose-response relations between sleep duration and risk for obesity.

24. Aziz M, Ali SS, Das S, Younus A, Malik R, Latif MA, et al. Association of subjective and objective sleep duration as well as sleep quality with non-invasive markers of sub-clinical cardiovascular disease (CVD): a systematic review. J Atheroscler Thromb. 2017;24(3):208-26.

25. Fatima Y, Doi SAR, Mamun AA. Longitudinal impact of sleep on overweight and obesity in children and adolescents: a systematic review and bias-adjusted meta-analysis. Obesity reviews: an official journal of the International Association for the Study of Obesity. 2015;16(2):137-49.

26. Chen X, Beydoun MA, Wang Y. Is sleep duration associated with childhood obesity? A systematic review and meta-analysis. Obesity. 2008;16(2):265-74. https://doi.org/10.1038/oby.2007.63.

27. Chaput J-P, Gray CE, Poitras VJ, Carson V, Gruber R, Olds T, et al. Systematic review of the relationships between sleep duration and health indicators in school-aged children and youth. Appl Physiol Nutr Metab. 2016;41(6 Suppl 3):S266-82.

28. Chaput JP, Gray CE, Poitras VJ, Carson V, Gruber R, Birken CS, et al. Systematic review of the relationships between sleep duration and health indicators in the early years (0-4 years). BMC Public 
Health. 2017;17(Suppl 5):855. https://doi.org/10.1186/s12889017-4850-2.

29. Fobian AD, Elliott L, Louie T. A systematic review of sleep, hypertension, and cardiovascular risk in children and adolescents. Curr Hypertens Rep. 2018;20(5):42. https://doi.org/10.1007/ s11906-018-0841-7.

30. Felso R, Lohner S, Hollody K, Erhardt E, Molnar D. Relationship between sleep duration and childhood obesity: systematic review including the potential underlying mechanisms. Nutr Metab Cardiovasc Dis. 2017;27(9):751-61. https://doi.org/10.1016/j. numecd.2017.07.008.

31. Jensen ME, Wood LG, Williams RB, Collins CE. Associations between sleep, dietary intake and physical activity in children: a systematic review. JBI Database System Rev Implement Rep. 2013;11(3):227-62.

32. Li L, Zhang S, Huang Y, Chen K. Sleep duration and obesity in children: a systematic review and meta-analysis of prospective cohort studies. J Paediatr Child Health. 2017.

33. Chiu HY, Lee HC, Chen PY, Lai YF, Tu YK. Associations between sleep duration and suicidality in adolescents: a systematic review and dose-response meta-analysis. Sleep Med Rev. 2018;42:11926. https://doi.org/10.1016/j.smrv.2018.07.003.

34. Buysse DJ, Reynolds CF 3rd, Monk TH, Berman SR, Kupfer DJ. The Pittsburgh Sleep Quality Index: a new instrument for psychiatric practice and research. Psychiatry Res. 1989;28(2):193-213. https://doi.org/10.1016/0165-1781(89)90047-4.

35. Nielsen LS, Danielsen KV, Sorensen TIA. Short sleep duration as a possible cause of obesity: critical analysis of the epidemiological evidence. Obes Rev. 2011;12(2):78-92.

36. Miller MA, Kruisbrink M, Wallace J, Ji C, Cappuccio FP. Sleep duration and incidence of obesity in infants, children, and adolescents: a systematic review and meta-analysis of prospective studies. Sleep. 2018;41(4). https://doi.org/10.1093/sleep/zsy018.

37. Van Dongen HPA, Maislin G, Mullington JM, Dinges DF. The cumulative cost of additional wakefulness: dose-response effects on neurobehavioral functions and sleep physiology from chronic sleep restriction and total sleep deprivation. Sleep. 2003;26(2):11726.

38. Dinges DF, Rogers NL, Baynard MD. Chronic sleep deprivation. Principles and practice of sleep medicine. 4th ed. Philadelphia, PA: WB Saunders Company; 2005. p. 67-76.

39. Wehr TA, Moul DE, Barbato G, Giesen HA, Seidel JA, Barker C, et al. Conservation of photoperiod-responsive mechanisms in humans. Am J Phys. 1993;265(4 Pt 2):846-57.

40. Short MA, Weber N, Reynolds C, Coussens S, Carskadon MA. Estimating adolescent sleep need using dose-response modeling. Sleep. 2018;41(4). https://doi.org/10.1093/sleep/zsy011 A clear description of dose-response effects of sleep restriction and extention in a clinical setting, analysed in a novel and promising method to determine sleep need in adolescents.

41. de Castro JM. The influence of heredity on self-reported sleep patterns in free-living humans. Physiol Behav. 2002;76(4-5):479-86. https://doi.org/10.1016/s0031-9384(02)00699-6.

42. Partinen M, Kaprio J, Koskenvuo M, Putkonen P, Langinvainio H. Genetic and environmental determination of human sleep. Sleep. 1983;6(3):179-85. https://doi.org/10.1093/sleep/6.3.179.

43. Watson NF, Buchwald D, Vitiello MV, Noonan C, Goldberg J. A twin study of sleep duration and body mass index. J Clin Sleep Med. 2010;6(1):11-7.
44. Meijer AM. Chronic sleep reduction, functioning at school and school achievement in preadolescents. J Sleep Res. 2008;17(4): 395-405.

45. Dewald JF, Short MA, Gradisar M, Oort FJ, Meijer AM. The Chronic Sleep Reduction Questionnaire (CSRQ): a cross-cultural comparison and validation in Dutch and Australian adolescents. J Sleep Res. 2012;21(5):584-94.

46. Medicine AAoS. International classification of sleep disorders, 3rd ed. Diagnostic and coding manual. Darien, IL: American Academy of Sleep Medicine; 2014.

47. de Bruin EJ, van Run C, Staaks J, Meijer AM. Effects of sleep manipulation on cognitive functioning of adolescents: a systematic review. Sleep Med Rev. 2017;32:45-57.

48. Lim J, Dinges DF. A meta-analysis of the impact of short-term sleep deprivation on cognitive variables. Psychol Bull. 2010;136(3):375-89.

49. Short MA, Blunden S, Rigney G, Matricciani L, Coussens S. C MR et al. Cognition and objectively measured sleep duration in children: a systematic review and meta-analysis. Sleep Health. 2018;4(3):292-300. https://doi.org/10.1016/j.sleh.2018.02.004.

50. Reynaud E, Vecchierini MF, Heude B, Charles MA, Plancoulaine S. Sleep and its relation to cognition and behaviour in preschoolaged children of the general population: a systematic review. J Sleep Res. 2018;27(3):e12636. https://doi.org/10.1111/jsr.12636.

51. Yeh AY, Pressler SJ, Giordani BJ, Pozehl BJ, Berger AM. Integrative review of the relationship between sleep disturbances and episodic memory in older adults. Biol Res Nurs. 2018;20(4): 440-51. https://doi.org/10.1177/1099800418768070.

52. Astill RG, Van der Heijden KB, Van Ijzendoorn MH, Van Someren EJW. Sleep, cognition, and behavioral problems in school-age children: a century of research meta-analyzed. Psychol Bull. 2012;138(6):1109-38.

53. Dewald JF, Meijer AM, Oort FJ, Kerkhof GA, Bogels SM. The influence of sleep quality, sleep duration and sleepiness on school performance in children and adolescents: a meta-analytic review. Sleep Med Rev. 2010;14(3):179-89.

54. Gregory AM, Sadeh A. Annual research review: sleep problems in childhood psychiatric disorders-a review of the latest science. J Child Psychol Psychiatry. 2016;57(3):296-317. https://doi.org/10. $1111 /$ jcpp. 12469.

55. Johnson EO, Roth T, Breslau N. The association of insomnia with anxiety disorders and depression: exploration of the direction of risk. J Psychiatr Res. 2006;40(8):700-8. https://doi.org/10.1016/j. jpsychires.2006.07.008.

56. Beattie L, Kyle SD, Espie CA, Biello SM. Social interactions, emotion and sleep: a systematic review and research agenda. Sleep Med Rev. 2015;24:83-100.

57. Lavie P. Self-reported sleep duration-what does it mean? J Sleep Res. 2009;18(4):385-6. https://doi.org/10.1111/j.1365-2869.2009. 00813.x.

58. Matricciani L. Subjective reports of children's sleep duration: does the question matter? A literature review. Sleep Med. 2013;14(4): 303-11.

Publisher's Note Springer Nature remains neutral with regard to jurisdictional claims in published maps and institutional affiliations. 